\title{
Photonic micro-resonators for steam evaporation dynamic sensing
}

\author{
Lucas Garnier ${ }^{1, *}$, Hervé Lhermite ${ }^{2}$, Véronique Vié ${ }^{1}$, Qingyue $\mathrm{Li}^{1}$, Mathieu Berges ${ }^{3}$, Vincent Cazin ${ }^{3}$, \\ Hervé Cormerais ${ }^{3}$, Jacques Weiss ${ }^{3}$, Etienne Gaviot ${ }^{4}$, and Bruno Bêche ${ }^{1, *}$ \\ ${ }^{1}$ Institut de Physique de Rennes, IPR CNRS 6251, Université de Rennes 1, 35042 Rennes, France \\ ${ }^{2}$ Institut d'Electronique et de Télécommunication de Rennes, IETR CNRS 6164, Université de Rennes 1, 35042 Rennes, France \\ ${ }^{3}$ CentraleSupelec, Campus de Rennes, 35510 Cesson-Sévigné, France \\ ${ }^{4}$ Laboratoire d'Acoustique de l'Université du Maine,LAUM CNRS 6313, Université du Maine, 72000 Le Mans, France
}

Received: 5 June 2018 / Received in final form: 14 September 2018 / Accepted: 5 October 2018

\begin{abstract}
We have investigated the effect of sudden water condensation processes and the behavior of its condensed water prior evaporation, with an integrated resonant photonic structure and dynamic tracking of its transduced signal. The aim of this analysis is to develop a water condensation lab-on-chip sensor, with the possibility of data treatment with an embedded system. Integrated photonic micro-resonator (MR) devices have been designed and fabricated with polymer UV210 by means of Deep-UV photolithography. Thanks to this technique, we have achieved racetrack shaped micro-resonators coupled to suited access waveguides. We have assessed such MRs with different geometrical characteristics while changing, respectively, the coupling length $\left(L_{\mathrm{C}}\right)$, the radius of curvature $(R)$ and the width $(w)$ of the guides. The chosen values for the set of parameters $L_{\mathrm{C}}-R-w($ in $\mu \mathrm{m})$ are 5-5-3 and 10-10-3. The laser source used with the injection bench is a Gaussian broadband laser $\left(\lambda_{\text {central }}=790 \mathrm{~nm}, \mathrm{FWHM}=40 \mathrm{~nm}\right)$ allowing us to visualize several resonances at the same time in order to multiplex the relevant measurements. The transduced spectrum is then acquired with an optical spectrum analyzer (OSA) linked to a computer with Labview and MATLAB software recording and processing data in real time. Then, relevant characteristics to be tracked are the Free Spectral Range (FSR) and the transmitted energy; these quantities can be linked to the physical characteristics of the structure considering both the effective refractive index and the absorption coefficient. The experimental setup also includes various movies with a top-view imaging camera of the chip (MRs) recording the soft matter process steps, so as to correlate the changes in the transduced spectrum and the behavior of the condensed water mechanisms (condensation, coalescence and evaporation). Then, the chip is fitted with a temperature controller, so as to carry out measurements at different temperatures: 20,24 and $28^{\circ} \mathrm{C}$.
\end{abstract}

\section{Introduction and background}

The accurate knowledge of humidity conditions in a gaseous environment is an important piece of information in numerous cases. Indeed, many industrial sectors need precise monitoring of the humidity in the air [1]. For example, in food industry, the conservation of edible products needs to be ensured with a well-controlled atmosphere in order to avoid the development of moisture. Various technical solutions may be considered to monitor humidity: capacitive hygrometers [2], resistive hygrometers [3], psychrometers [4,5] and dew-point hygrometers and so on. Technical solutions to monitor humidity via photonic structures are already investigated by several research teams [6]. In this article, the interest is focused on the possibility to measure humidity with integrated photonic micro-resonators by studying the influence of water condensation and evaporation on the mode propagating in such photonic structures. Photonic

\footnotetext{
* e-mail: lucas.garnier@univ-rennes1.fr
}

micro-resonator sensors have known a large expansion in the past few years [7]. Indeed, specific properties of these devices foster significant advantages compared to many other sensing techniques. The integration of such sensors on relevant chips allows designing lab-on-chip sensors [8], which facilitate both miniaturization and transportability [9]. This kind of structure also provides very precise measurements, with low limit of detection and high sensitivity [10]. As miniaturizable and most accurate, photonic micro-resonator sensors come up as very efficient in following small-scale phenomena or in detecting sparsely concentrated chemical species [11]. These characteristics are most interesting for both environmental monitoring [12] and biology together with biomedical or biophysics [13] fundamental research (among other fields). However, with a large majority of scientific works using integrated micro-resonator sensors focusing on static phenomena [9,11-13], most often the transduced signal of the photonic structure is monitored against concentration values of given species or versus temperature. Then, noteworthy variations of the signal versus time, so as to measure dynamic reactions, are rarely 
investigated. In this paper, integrated micro-resonators are used to perform a dynamic tracking of water condensation and evaporation mechanisms on the acting surface of the sensor.

The transduced signal of a micro-resonator coupled to an access waveguide depends on two phenomena. The first one is the coupling which is an optic tunnel effect between the access waveguide and the resonator. The optogeometric parameters of the structure determine the quality of the coupling. The second phenomenon is the resonance caused by the excitation of stationary modes in the resonator. This phenomenon is driven, for a given geometry, by the refractive index of the surrounding medium. The transfer function associated to a microresonator coupled to an access waveguide is given in reference [14]:

$$
T(\Phi)=(1-\gamma)\left[1-\frac{\left(1-a^{2}\right)\left(1-\tau^{2}\right)}{(1-a \tau)^{2}+4 a \tau \sin ^{2}\left(\frac{\Phi}{2}\right)}\right],
$$

where $\Phi=\beta P$, the phase delay inside the resonator for a single trip (with $\beta$ the propagation constant of the mode and $P$ the opto-geometrical perimeter), $\gamma$ is the intensityinsertion-loss coefficient, $\tau$ is the internal amplitude transmission coefficient which corresponds to the quantity of energy that is not coupled from the access waveguide to the resonator and vice versa. Finally, $a=\sqrt{1-\gamma} e^{-\rho L_{m c}}$ is the total amplitude loss factor, related to $\rho$ the amplitude attenuation coefficient in the resonator and $L_{\mathrm{mc}}$ the mean circumference of the resonator excluding the coupling part. These different parameters $(\beta, \tau, \gamma, \rho$ and $a)$ are suddenly perturbed by the condensation of water and continuously vary during its evaporation. The measurable spectral characteristics that can be related to these parameters are the quality factor $Q$, the finesse $F$, the full width at half maximum FWHM and the free spectral range (FSR). Each one of these quantities strongly depends on several of the above parameters, except the FSR, that only depend on the effective refractive index $n_{\text {eff }}^{g}=\frac{\beta}{k}$, where $k$ is the wavenumber associated with the mode. As the refractive index of water is superior to the index of air, the effective index of the mode is correlated to the presence or not of condensed water on the sensor. The relevant parameter to track is in the free spectral range, i.e. the distance between two consecutive resonances in the spectral domain. The FSR is given in reference [15]:

$$
\mathrm{FSR}=\frac{\lambda_{0}^{2}}{P n_{\mathrm{eff}}^{g}},
$$

where $\lambda_{0}$ is the central wavelength, $P$ the opto-geometrical perimeter of the resonator and $n_{\text {eff }} \mathrm{g}$ the effective group refractive index associated to the mode, which depends on the optical index of both the waveguide itself and its close environment. By tracking the FSR, we are so able to track any change in the refractive index above the resonator.

Water condensation on a surface depends on the relative humidity $(\mathrm{RH})$ of its surrounding environment. The quantity $\mathrm{RH}$ is defined as the ratio between the partial pressure of water vapor $\mathrm{p}_{\mathrm{H} 2 \mathrm{O}}$ and the saturation vapor pressure (in equilibrium) noted $p_{\mathrm{e}}: R H_{\%}=100 \cdot p_{p_{\mathrm{H}} \mathrm{O}}$. The saturation vapor pressure depends on the temperature of the air: the greater the temperature, the higher the equilibrium vapor pressure. The condensation occurs when the $\mathrm{RH}$ value reaches $100 \%$. As the $\mathrm{RH}$ increases when temperature decreases (because $p_{\mathrm{e}}$ decreases), the condensation occurs when the air is cooled down (until $p_{e}=p_{\mathrm{H}_{2} \mathrm{O}}$ ): for example, during the night, in humid regions, dew appears when the temperature becomes sufficiently low (reaching the so-called dew point $t_{\mathrm{d}}$ ) or when the air is in contact with a cold surface (with a temperature $t<t_{\mathrm{d}}$ ).

In the second section, the fabrication of the structures is detailed. The experimental setup is presented in the third section, with relevant results exposed and discussed in Section 4.

\section{Structure fabrication}

\subsection{Materials}

In the literature, two types of materials are mainly used for the fabrication of integrated photonic structures: semiconductors and polymers [15]. In this study, the chosen material is the polymer DUV210 [16], for the following reasons: first, refractive indices of polymers are smaller than the ones of semiconductors; for an upper cladding composed of air or water, which is often the case for sensing applications, the evanescent wave of the propagating mode is then larger for polymer resonators than for semiconductor structures. This difference implies better sensing properties for polymers based on resonators. Secondly, polymers are quite less expensive than semiconductors, reducing then the fabrication cost and facilitating industrial large-scale production. Eventually, among other polymers, we have selected the DUV210 as a chemically amplified positive resin due to its specific absorption band at $248 \mathrm{~nm}$ [17], allowing performing deep-UV photolithography most suited to design submicronic gaps. An advantageous substrate for such structures is a $\mathrm{SiO}_{2} / \mathrm{Si}$ wafer, with a $\mathrm{SiO}_{2}$ layer $1 \mu \mathrm{m}$ in thickness obtained by thermal oxidation. This layer stands as the lower cladding for the waveguides.

\subsection{Geometrical characteristics}

Beyond the choice of the material, the shape and the geometrical characteristics of the structure are determinant factors with a view to sensing properties. The structure hinges on a mono-mode access waveguide laterally coupled from either side to four identical mono-mode racetrack shaped micro-resonators as depicted in Figure 1. The monomode nature of these structures has first been confirmed in a theoretical way by a FDTD simulation of the propagation of the optical power inside the overall structure, then in an experimental way by imaging both the cross-sectional output by the means of a CCD camera and the top of the chip under injection. The presence of multiple resonators leads to a greater extinction ratio and quality factor, and allow more precise measurements [18]. The resonators are characterized by four quantities: the coupling length $L_{\mathrm{C}}$, the radius of curvature $R$ associated with the bent part of the 


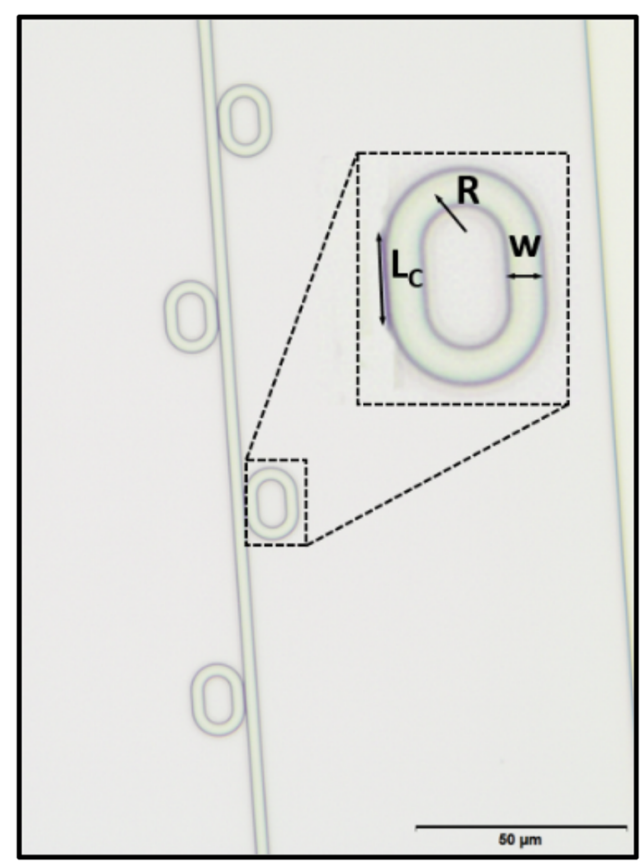

Fig. 1. Optical imaging of the photonic structure.

resonator, the gap $g$ between the access waveguide and the resonator and the width $w$, made equal for each resonator and its access waveguide as shown in Figure 2. The study has been performed on structures with $L_{\mathrm{C}^{-}} R$ - $w$ set values equal to 5-53 and 10-10-3 in micrometers ( $g=0.4 \mu \mathrm{m}$ for each) in order to estimate the impact of the size of a resonator on its sensing properties. Using lateral coupling allows fabricating the chip by way of a single deep-UV insolation, reducing then the fabrication costs and allowing eradication of any misalignment hazard in case of necessary multiple insolation.

\subsection{Fabrication process}

The structures have been fabricated in a class 100 cleanroom. The wafer supporting the photonic circuits is a silicon wafer on which has been performed a thermal oxidation to obtain a $\mathrm{SiO}_{2}$ layer of $1 \mu \mathrm{m}$. The UV210 is spread on the wafer by spin coating, with the relevant speed and acceleration values in order to get a 800 -nm thick layer. This thickness is necessary to make all the structures monomode. The sample is then exposed to deep-UV via a mercury lamp to reproduce structure's shapes previously designed on a photolithography mask. Then, development is ensured with tetramethylammonium hydroxide (TMAH). Each one of these steps is followed by a softbake to consolidate the structure. A quality control is then performed on these structures to verify their geometrical characteristics such as the width of the guides as well as the gap separating the access waveguide and the resonators.

\section{Experimental setup}

So as to carry out the measurements, the resonators are placed on an injection bench as depicted in Figure 3. The laser source used in this bench is a Gaussian broadband

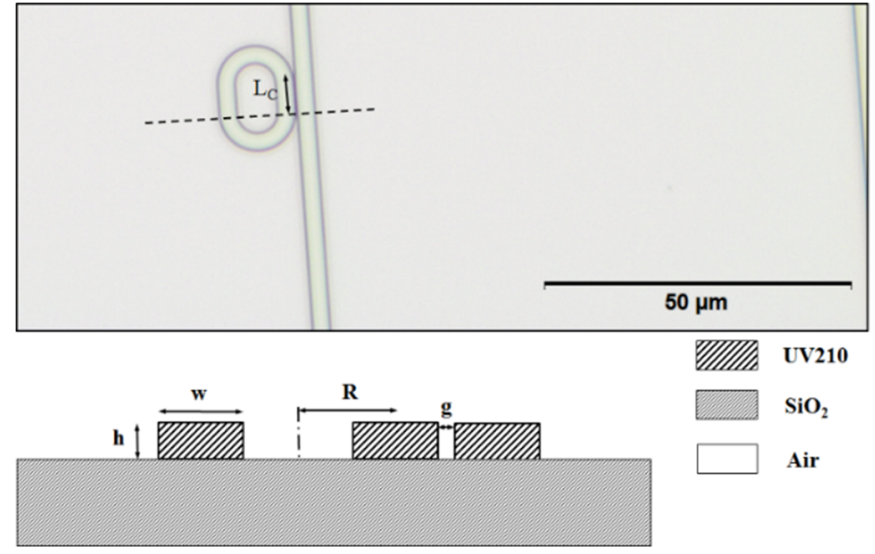

Fig. 2. Optical top-view imaging and schematic sectional view of the photonic device.

laser diode with $\lambda_{0}=790 \mathrm{~nm}$ central wavelength and $\mathrm{FWHM}=40 \mathrm{~nm}$. The light is then TE-polarized before being injected in the access waveguide in order to excite only the $\mathrm{TE}_{00}$ mode. The latter operation is performed by way of microscope objectives focusing the light within the guide, and defocusing it after proceeding through the photonic structure. Any resulting ray of light is then split by a beamsplitter prior respective analysis with a CCD camera (PulNix CCD camera) and an optical spectrum analyzer (OSA Ando AQ-6315E). A top-view imaging camera is placed above the chip to link the spectral results to a visual of the process. The width of the optical spectrum produced by the laser allows us to visualize several resonances together with each spectrum acquired by the OSA. The OSA is remotely controlled by a MATLAB software $^{1}$ which allows defining every parameter of the acquisition (span, resolution, etc.). A program has also been implemented for both the acquisition of the spectra and the data treatment in real time. The FSR is extracted from each spectrum by computing its Fast Fourier Transform (FFT) and extracting its maximum value by a Lagrange polynomial interpolation. Finally, the photonic circuit is thermally controlled so as to quantify the influence of temperature on the behavior of this circuit, while a top-view imaging camera allows correlating the steam behavior with the modifications in the transduced optical signal.

\section{Results and discussions}

Once the setup is ready, the spectra are continuously acquired. A few seconds after the beginning of the acquisition, the water condenses on the sensor. The water condensation is obtained by blowing a hot flow of humid air; its contact with a cooler surface (the chip) leads to the condensation of part of the water contained in this air stream. The $\mathrm{RH}$ of the room we perform the experiments in is approximately $50 \%$. After whole evaporation of the water, acquisition is carried further out for a short amount of time to

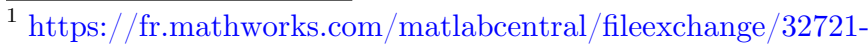
getosatrace
} 


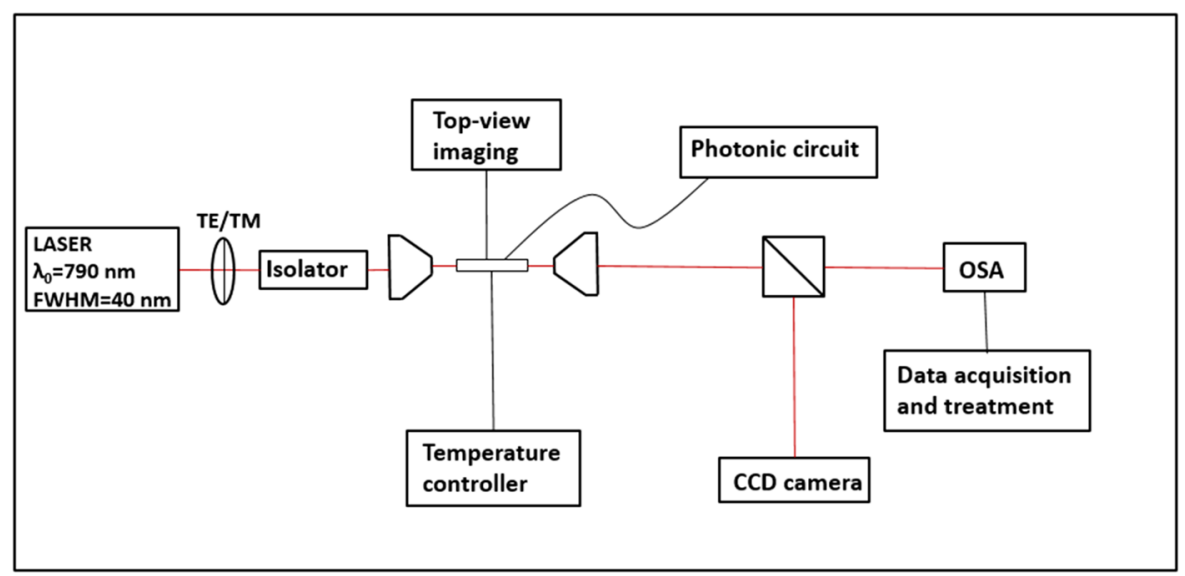

Fig. 3. Schematic representation of the experimental setup; the acquisition and treatment of data is made in real time.

a)

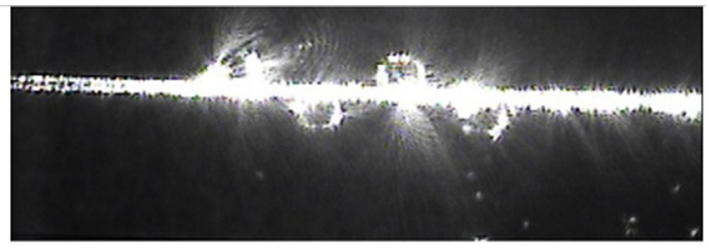

b)

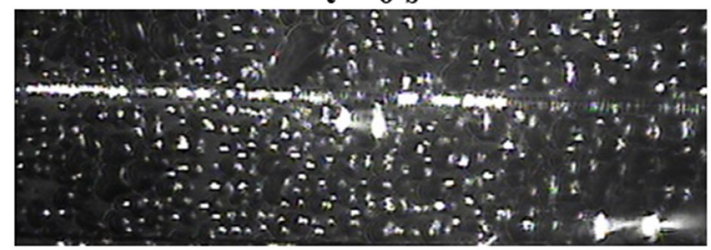

c)

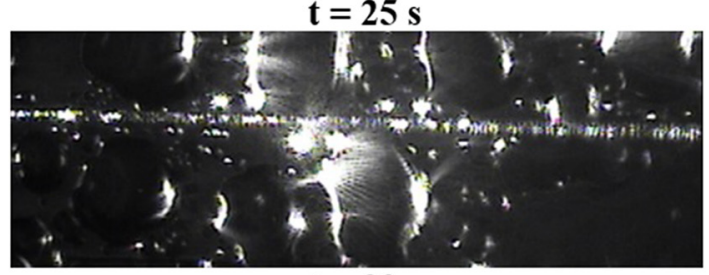

$\mathrm{t}=\mathbf{3 0} \mathrm{s}$

d)

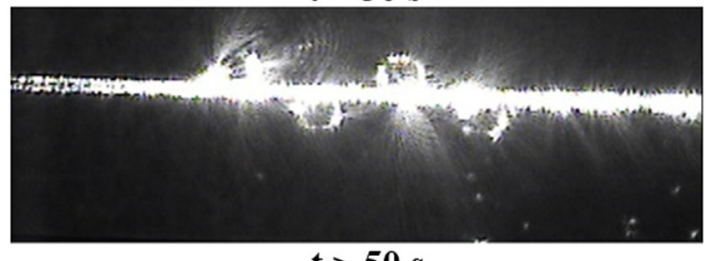

$\mathbf{t}>\mathbf{5 0} \mathrm{s}$

Fig. 4. Top-view imaging of the chip under injection during the condensation/evaporation process at (a) $t=0 \mathrm{~s}$, (b) $t=25 \mathrm{~s}$, (c) $t=30 \mathrm{~s}$ and (d) $t>50 \mathrm{~s}$.

ensure the resonator being reset back to its initial state. Figure 4 shows photos extracted from a movie of the surface of the chip at different times, corresponding to different phases in the condensation/evaporation process. Figures $4 \mathrm{a}$ and $4 \mathrm{~d}$ correspond to initial and final state, i.e. before the water condenses and after it evaporates. Figures $4 \mathrm{~b}$ and $4 \mathrm{c}$ show the condensed water before and after coalescence, respectively. When water condenses on a surface, small drops appear; the coalescence phenomenon is the fusion of several of these small drops into bigger ones in order to decrease the surface energy and reach a more stable configuration. This coalescence phenomenon occurs to rapidly be investigated with our setup; the present study focuses on evaporation dynamics. Figure 5 represents spectra at different times during this process, for two different mono-mode resonant structures. This graph yields that, considering mono-mode resonators, the greater the perimeter of the resonator, the smaller the free spectral range as predicted in equation (2).

For each spectrum, the FSR and the maximum intensity are extracted by means of an automatized data treatment program. Evolution of these characteristics over time is depicted in Figure 6.

A strong correlation comes up between the FSR and the maximum intensity, owing to the fact that as water condenses on the sensor, the upper cladding of the photonic structure changes from air to condensed water. This upper cladding change yields two major effects on the intensity. Firstly, as the cladding index changes, the acceptance cone of the access waveguide is also modified, leading to a lower coupling strength between the laser ray and the access waveguide, with then a diminished amount of optical power in the structure. Secondly, as the cladding index is increased, the evanescent wave of the propagating mode enlarges itself and is partly absorbed by the condensed water. Intensity variations are also confirmed by the photos in Figure 4. Such effects being difficult to quantify and decorrelate, the present study is focused on the FSR dynamics. The graphs representing the evolution of the FSR can be sliced in four zones (A, B, C and D) as shown in Figure 6a. Zones A and D correspond to the bare sensor, i.e. a resonator with air as upper cladding. In zone B, as the water has just condensed, the amount of water is sufficient to form a semi-infinite cladding; hence, as long as the amount of water is sufficient, the FSR does not vary. This value of the FSR corresponds to the test value of these structures when the upper cladding is entirely composed of water. This situation is equivalent to the spontaneous condensation of water in case relative humidity is $100 \%$. Finally, in Zone C, the water is progressively evaporating, yielding the FSR to decrease until reaching again its initial value when the water is entirely evaporated. Figure 7 shows the schematic representation of this process accomplished in four steps. 
a)

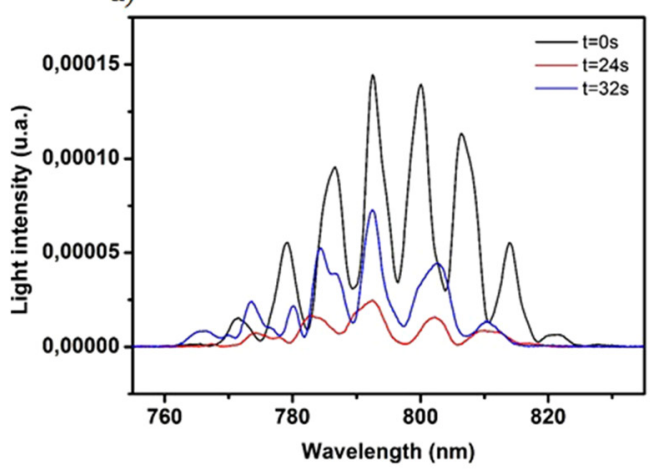

b)

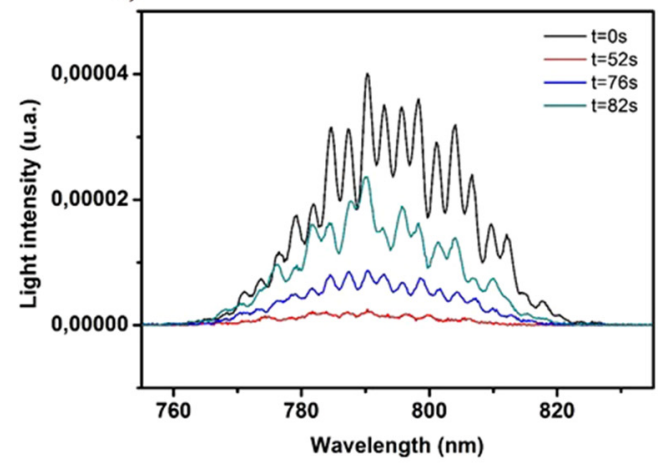

Fig. 5. Examples of spectra acquired at different times during the process for values $L_{\mathrm{C}^{-}} R-w$ of (a) $5-5-3$ (b) $10-10-3$ in micrometers.
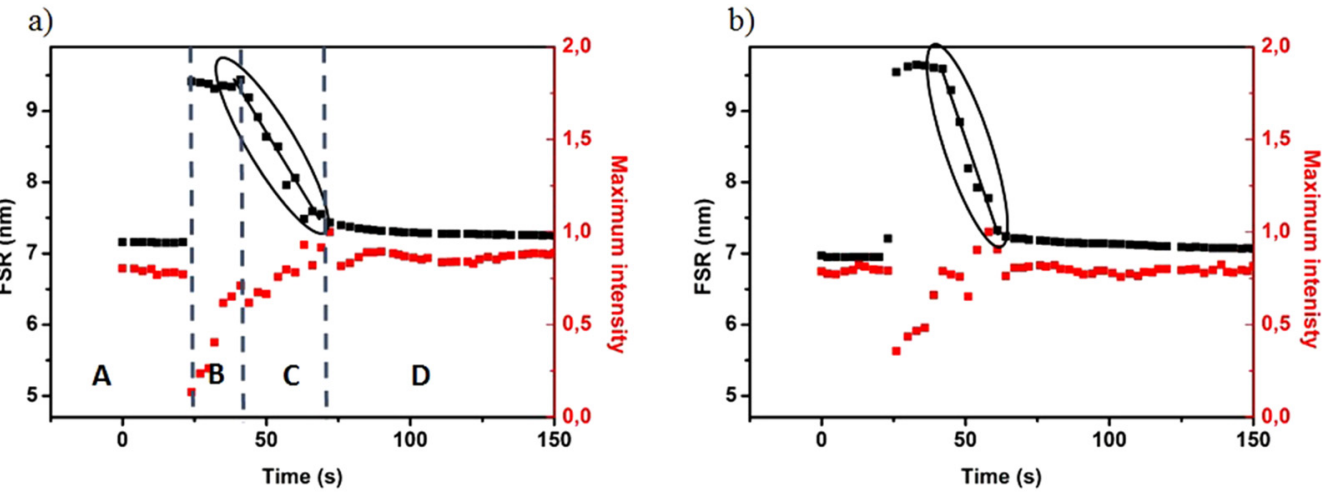

Fig. 6. Evolution of the free spectral range (black) and of the maximum intensity (red) for (a) $5-5-3$ structure, at $T=\left(\right.$ a) $20^{\circ} \mathrm{C}$ and (b) $28^{\circ} \mathrm{C}$.

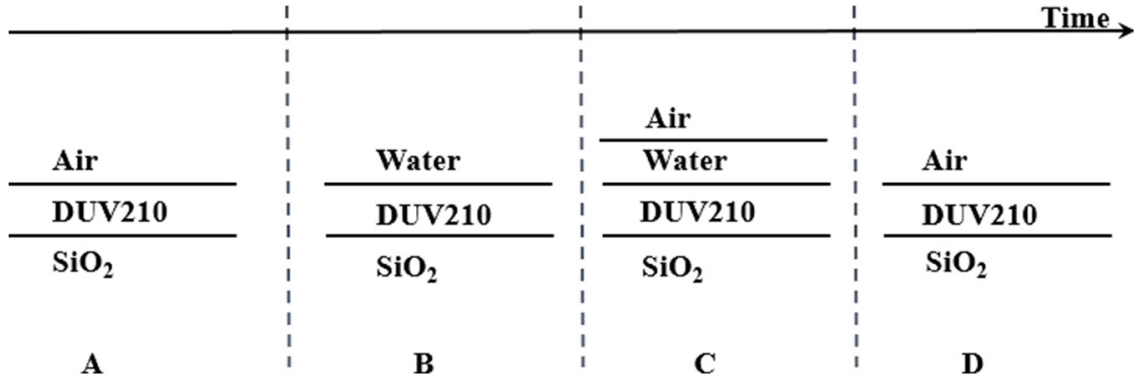

Fig. 7. Schematic representation of the succession of the different upper cladding for each zone.

The principal interest of this study relies in the zone C, considering particularly the slope of the linear regression of the dynamics of the FSR $\frac{d F S R}{d t}$, quantity closely related to the evaporation kinetics. Then, Table 1 summarizes the results for both geometrical configurations as regard different temperatures.

In order to get rid of the influence of the size of the resonator on the slope, the above values are normalized by the initial value of the FSR, i.e. the value before the condensation of water, with air as upper cladding. These values are presented on Table 2 . These results show that the higher the temperature, the steeper the slope due to the evaporation speed increased with temperature. The differences between the 5-5-3 and the 10-10-3 structures are due to the value of the radius of curvature $R$. Indeed, the smaller the radius of curvature, the larger the evanescent wave, leading then to increased variations of the FSR. We have thus proved that fixed geometrical parameters $\left(L_{\mathrm{C}}-R-w\right)$ imply a temporal sensibility to FSR variations.

The possibilities to design humidity sensors from this optical integrated device are of two kinds. On the one hand, the difference between Zones A and B allows designing a dew-point sensor, which can detect the presence or not of condensed water on the surface of such a sensor. In the 
Table 1. Numerical values of $\frac{d F S R}{\mathrm{~d} t}(\mu \mathrm{m} / \mathrm{s})$ for different sensing configurations.

\begin{tabular}{lllr}
\hline Resonators $L_{c}-R-w(\mu \mathrm{m})$ & \multicolumn{2}{c}{$T\left({ }^{\circ} \mathrm{C}\right)$} \\
\cline { 2 - 4 } & 20 & 24 & 28 \\
\hline $5-5-3$ & -0.067 & -0.075 & -0.110 \\
$10-10-3$ & -0.011 & -0.022 & -0.039 \\
\hline
\end{tabular}

Table 2. Normalized values of $\frac{d F S R}{d t}(\mu \mathrm{m} / \mathrm{s})$ with respect to nominal FSR values.

\begin{tabular}{lllr}
\hline Resonators $L_{c}-R-w(\mu \mathrm{m})$ & \multicolumn{2}{c}{$T\left({ }^{\circ} \mathrm{C}\right)$} & 28 \\
\cline { 2 - 4 } & 20 & 24 & -0.016 \\
\hline $5-5-3$ & -0.008 & -0.010 & -0.014 \\
$10-10-3$ & -0.004 & -0.008 & \\
\hline
\end{tabular}

other hand, Zone $\mathrm{C}$ yields further information. As we have seen previously, the slope $\frac{d F S R}{\mathrm{~d} t}$ in Zone $\mathrm{C}$ is related to evaporation dynamic process, which is physically representative of the ability of the surrounding air (considered as a reservoir of gas at fixed relative humidity) to exchange mass of water with the liquid medium. No exact quantitative analytical link between these two quantities is presented in the literature, but the present study clearly highlights their interdependency. The evaporation speed, as for it, is directly correlated to the relative humidity for given conditions of temperature and pressure. By performing a proper calibration in controlled conditions of humidity, temperature and pressure, it is thus possible to design a relative humidity sensor by an adequate monitoring of water evaporation conditions.

\section{Conclusion}

This work presents a quantitative study about the behavior of integrated photonic micro-resonators subject to sudden water condensation. By dynamically tracking the free spectral range of the resonant structure against time, we have pointed out four steps during the condensation/ evaporation process, among which one is correlated to the evaporation dynamics. These measurements have been realized on two different geometrical structures characterized by their values of coupling length and radius of curvature, and for three different temperatures. By performing a proper calibration of such a device, the design of a humidity sensor is possible. Moreover, the UV-210 material and the single deep-UV photolithography used for the fabrication of this lab-on-chip sensor allow a low-cost production of such devices. In this study, the interest is focused on the analysis of the temporal variations of the free spectral range, but to achieve a complete description of the phenomenon of condensation/ evaporation process, wider investigations should be undertaken on the other characteristics of the structures, such as the $Q$ factor and the finesse. These investigations would give more information on the dynamics of the coupling conditions between the access waveguide and the resonators.

\section{Author contribution statement}

Garnier performed all the experiments along with $\mathrm{Li}$, and he drafted the manuscript. Lhermite worked on the fabrication of the chip device. Berges, Cazin, Cormerais and Weiss programmed the data treatment software. Vié and Gaviot brought the interpretation concerning fluidic and thermal considerations, respectively. Bêche supervised the project and worked on the manuscript. All authors discussed the results and approved the final manuscript.

\section{References}

1. G. Asch, B. Poussery, Les capteurs en instrumentation industrielle, 8th edn (Dunod, Paris, 2017)

2. P. Neis, H.G.J. Smit, S. Rohs, U. Bundke, M. Krämer, N. Spelten, V. Ebert, B. Buchholz, K. Thomas, A. Petzold, Tellus B Chem. Phys. Meteorol. 67, 28320 (2015)

3. K.-P. Yoo, L.-T. Lim, N.-K. Min, M.J. Lee, C.J. Lee, C.-W. Park, Sens. Actuators B 145, 120-125 (2010)

4. N. Giordani, L. Camberlein, E. Gaviot, F. Polet, N. Pelletier, B. Bêche, IEEE Trans. Instrum. Measure. 56, 102-106 (2007)

5. Z. Chen, C. Lu, Sens. Lett. 3, 274-295 (2005)

6. Q. Wu, Y. Semenova, J. Mathew, P. Wang, G. Farrell, Opt. Lett. 36, 1752-1754 (2011)

7. I. Chremmos, O. Schwelb, N. Uzunoglu, Photonic Microresonator Research and Applications, Optical Sciences Series (Springer, Berlin, 2010)

8. C.F. Carlborg, K.B. Gylfason, A. Kazmierczak, F. Dortu, M.J. Banuls Polo, A. Maquieira Catala, G.M. Kresbach, H. Sohlström, T. Moh, L. Vivien, J. Popplewell, G. Ronan, C.A. Barrios, G. Stemme, W. van der Wijngaart, Lab Chip 10, 257-393 (2010) 
9. L. Gounaridis, P. Groumas, E. Schreuder, G. Tsekenis, A. Marousis, R. Heidman, H. Avramopoulos, C. Kouloumentas, Opt. Express 25, 7483-7495 (2017)

10. C.-Y. Chao, W.L. Jay Guo, IEEE J. Sel. Top. Quantum Electron. 12, 134-142 (2006)

11. R. Castro-Beltràn, N. Huby, V. Vié, H. Lhermite, L. Camberlein, E. Gaviot, B. Bêche, Adv. Device Mater. 1, 80-87 (2015)

12. F. Meziane, V. Raimbault, H. Hallil, S. Joly, V. Conédéra, J.L. Lachaud, L. Béchou, D. Rebière, C. Dejous, Sens. Actuators B 209, 1049-1056 (2015)

13. Q. Li, V. Vié, H. Lhermite, E. Gaviot, C. Bourlieu, A. Moréac, D. Morineau, D. Dupont, S. Beaufils, B. Bêche, Sens. Actuators A 263, 707-717 (2017)

14. C. Delezoide, Ph.D thesis, Ecole Normale Supérieure de Cachan, 2012
15. D.G. Rabus, Integrated Ring Resonators, Optical Sciences Series (Springer, Berlin, 2007)

16. M. Specht, N. Huby, H. Lhermite, R. Castro-Beltràn, G. Loas, B. Bêche, Eur. Phys. J. Appl. Phys. 71, 10501 (2015)

17. D. Duval, H. Lhermite, C. Godet, N. Huby, B. Bêche, J. Opt. 12, 055501/1-6 (2010)

18. R. Castro-Beltràn, N. Huby, G. Loas, H. Lhermite, D. Pluchon, B. Bêche, J. Micromech. Microeng. 24, 125006 (2014)

Open Access This article is distributed under the terms of the Creative Commons Attribution License https://creativecom mons.org/licenses/by/4.0 which permits unrestricted use, distribution, and reproduction in any medium, provided the original author(s) and source are credited.

Cite this article as: Lucas Garnier, Hervé Lhermite, Véronique Vié, Qingyue Li, Mathieu Berges, Vincent Cazin, Hervé Cormerais, Jacques Weiss, Etienne Gaviot, Bruno Bêche, Photonic micro-resonators for steam evaporation dynamic sensing, Eur. Phys. J. Appl. Phys. 84, 10502 (2018) 